\title{
EVIDENCE FOR MODIFIED TRANSPORT DUE TO SHEARED E X B FLOWS IN HIGH-TEMPERATURE PLASMAS
}

\author{
by
}

R.J. GROEBNER, M.E. AUSTIN, K.H. BURRELL, T.N. CARLSTROM, S. CODA, E.J. DOYLE, P. GOHIL, J. KIM, R.J. LA HAYE, C.L. HSIEH, L.L. LAO, J. LOHR, R.A. MOYER, C.L. RETTIG, T. RHODES, G.M. STAEBLER, R.E. STOCKDALE, D.M. THOMAS, and J.G. WATKINS

This report was prepared as an account of work sponsored by an agency of the United States Government. Neither the United States Government nor any agency thereof, nor any of their employees, makes any warranty, express or implied, or assumes any legal liability or responsibility for the accuracy, completeness, or usefulness of any information, apparatus, product, or process disclosed, or represents that its use would not infringe privately owned rights. Reference herein to any specific commercial product, process, or service by trade name, trademark, manufacturer, or otherwise does not necessarily constitute or imply its endorsement, recommendation, or favoring by the United States Government or any agency thereof. The views and opinions of authors expressed herein do not necessarily state or reflect those of the United States Government or any agency thereof. 


\section{DISCLAIMER}

Portions of this document may be illegible in electronic image products. Images are produced from the best available original document. 


\title{
EVIDENCE FOR MODIFIED TRANSPORT DUE TO SHEARED E X B FLOWS IN HIGH-TEMPERATURE PLASMAS
}

\author{
by
}

R.J. GROEBNER, M.E. AUSTIN, ${ }^{*}$ K.H. BURRELL, T.N. CARLSTROM, S. CODA, $†$ E.J. DOYLE, † P. GOHIL, J. KIM, R.J. LA HAYE, C.L. HSIEH, L.L. LAO, J. LOHR, R.A. MOYER, $\ddagger$ C.L. RETTIG, $\ddagger$ T. RHODES $\ddagger$ G.M. STAEBLER, R.E. STOCKDALE, D.M. THOMAS, and J.G. WATKINSI

This is a preprint of a paper to be presented at the International Conference on Plasma Physics, October 31-November 4, 1994, Foz do Iguacu, Brazil, and to be printed in the Proceedings.

Work supported by U.S. Department of Energy

Contract Nos. DE-AC03-89ER51114, W-7405-ENG-48, DE-AC05-840R21400, DE-AC03-76DP00789, and Grant Nos. DE-FG03-89ER52212, DE-FG03-86ER53225, and DE-FG02-91ER54109

*University of Maryland

tMassachusetts Institute of Technology

fUniversity of California at Los Angeles

ISandia National Laboratories

\section{GENERAL ATOMICS PROJECT 3466 NOVEMBER 1994}




\title{
EVIDENCE FOR MODIFIED TRANSPORT DUE TO SHEARED EXB FLOWS IN HIGH-TEMPERATURE PLASMAS
}

\author{
R.J. Groebner, M.E. Austin, ${ }^{(a)}$ K.H. Burrell, T.N. Carlstrom, S. Coda, ${ }^{(b)}$ E.J. Doyle, ${ }^{(c)}$ \\ P. Gohil, J. Kim, R.J. La Haye, C.L. Hsieh, L.L Lao, J. Lohr, R.A. Moyer, ${ }^{(c)}$ \\ C.L. Rettig, ${ }^{(c)}$ T. Rhodes ${ }^{(c)}$ G.M. Staebler, R.E. Stockdale, D.M. Thomas, \\ and J.G. Watkins ${ }^{(d)}$
}

\author{
General Atomics, P.O. Box 85608, San Diego, California 92186-9784 USA \\ ${ }^{(a)}$ University of Maryland, USA \\ (b) Massachusetts Institute of Technology, USA \\ (c) University of California at Los Angeles, USA \\ ${ }^{(d)}$ Sandia National Laboratories, Albuquerque USA
}

\begin{abstract}
Sheared mass flows are generated in many fluids and are often important for the dynamics of instabilities in these fluids. Similarly, large values of the $E \times B$ velocity have been observed in magnetic confinement machines and there is theoretical and experimental evidence that sufficiently large shear in this velocity may stabilize important instabilities. Two examples of this phenomenon have been observed in the DIII-D tokamak. In the first example, sufficient heating power can lead to the L-H transition (transition from low-mode to high-mode confinement), a rapid improvement in confinement in the boundary layer (narrow region just inside the last closed flux surface) of the plasma. For discharges with heating power close to the threshold required to get the transition, changes in the edge radial electric field are observed to occur prior to the transition itself. In the second example, certain classes of discharges with toroidal momentum input from neutral beam injection exhibit a further improvement of confinement in the plasma core leading to a regime called the $\mathrm{VH}$-mode. In both examples, the region of improved confinement is characterized by an increase of shear in the radial electric field $E_{\mathbf{r}}$, reduced levels of turbulence and increases in gradients of temperatures and densities. These observations are consistent with the hypothesis that the improved confinement is caused by an increase in shear of the $\mathrm{E} \times \mathrm{B}$ velocity which leads to a reduction of turbulence. For the $\mathrm{VH}$-mode, the dominant term controlling $E_{\mathbf{r}}$ is the toroidal rotation $v_{\phi}$, indicating that the $E_{\mathrm{r}}$ profile is controlled by the source and transport of toroidal momentum. At the edge of the plasma, indirect measurements indicate that the change in $E_{\mathbf{r}}$ is initiated by a change in the $\mathrm{V} \times \mathrm{B}$ velocity of the main ions but at later times is dominated by the ion diamagnetic velocity.
\end{abstract}

\section{INTRODUCTION}

It has long been recognized in fluid mechanics that the interaction between sheared velocity fields and turbulence can have important consequences for the stability of various modes. Sufficiently large velocity shear can drive the Kelvin-Helmholtz mode unstable [1]. On the other hand, Rayleigh-Taylor modes can be stabilized by velocity shear [2]. Theoretical and experimental work during the last few years has provided strong evidence that similar mechanisms are important in magnetized plasmas. For this case, the fundamental velocity is not the mass velocity but is rather the $E \times B$ velocity, the velocity at which turbulent eddies are convected, where $\mathrm{E}$ and $\mathrm{B}$ are the electric and magnetic fields. Basic scaling arguments of nonlinear theory show that if the shear (spatial gradient) of the $E \times B$ velocity varies sufficiently on the scale of a turbulent eddy, the correlation time of the eddy is decreased by the shear [3]; thus, the eddy is effectively ripped apart by the 
shear leading to a reduction of the rate of transport due to turbulent processes. Numerous linear stability calculations have shown that sufficient shear in the $\mathrm{E} \times \mathrm{B}$ velocity is a robust mechanism to stabilize flute-like modes [4] (modes whose structures are elongated along magnetic field lines). Self-consistent nonlinear simulations have been performed for several modes of interest and they generally support the results of the linear analyses [5]. An important difference is that for some modes, the curvature (second spatial derivative) of the $E \times B$ velocity field is predicted to me much more important for stabilizing turbulence than the shear (first spatial derivative) [6].

There is a significant body of experimental evidence that sheared $E \times B$ flows do in fact modify and reduce the transport in magnetic confinement devices [7-9]. The evidence comes from machines with a variety of magnetic geometries and indicates that the effect is a basic phenomenon of magnetized plasmas rather than a peculiarity of a given confinement scheme. The clearest example in tokamaks is the $\mathrm{L}-\mathrm{H}$ transition, which is a spontaneous transition from a base level of confinement called the L-mode to an increased level of confinement called the $\mathrm{H}$-mode [10]. The transition to $\mathrm{H}-$ mode is initiated in a narrow layer at the periphery of the plasma. In this narrow layer, it is simultaneously observed that large shear (both first and second spatial derivatives) develops in the radial electric field $E_{\mathbf{r}}$, that the level of density fluctuations decreases, and that temperature and density gradients increase. This phenomenology is consistent with, and in some cases has been predicted by, the theoretical work on suppression of turbulence due to sheared $\mathrm{E} \times \mathrm{B}$ flows. Thus, the theoretical paradigm that shear in the $\mathrm{E} \times \mathrm{B}$ flow can stabilize turbulence has become the primary hypothesis for guiding studies of the L-H transition. Additional support is given to the hypothesis by experiments in which the transition has been induced with application of a radial electric field from a probe inserted part way into the plasma [11].

In at least two tokamaks, DIII-D [12] and JET [13], the H-mode discharge has been observed to evolve into a further improved regime of confinement called the VH-mode, which exhibits values of $\tau_{\mathrm{E}}$ which are 3-4 times those in L-mode discharges. In DIII-D, the phenomenology of the $\mathrm{VH}$-mode is also consistent with the hypothesis of $\mathrm{E} \times \mathrm{B}$ shear suppression of turbulence $[14,15]$. Whether this phenomenology is peculiar to DIII-D or is a universal aspect of the $\mathrm{VH}$-mode remains to be seen.

The $E \times B$ shear suppression mechanism is of interest for both basic and applied studies of plasma physics. Non-linear analyses show that a fundamental understanding of saturated turbulence in a magnetized plasma requires self-consistent knowledge of both $E \times B$ suppression [16] and transport of momentum by the turbulent fields [17]. From a practical point of view, design studies aimed at producing a tokamak which can obtain an ignited thermonuclear burn generally indicate that some enhancement of confinement over $\mathrm{L}$-mode levels is required to build a practical machine. $\mathrm{H}$-mode and VH-mode discharges are candidates for achieving this increased confinement. Thus, if $E \times B$ shear suppression is required to produce the $\mathrm{H}$-mode and $\mathrm{VH}$-mode plasmas, it is a rather important subject from the point of view of applied physics as well as basic physics.

The goal of this paper is to examine the present status of $\mathrm{H}$-mode and VH-mode studies in the DIII-D tokamak. In Section II, the phenomenology of the L-H transition and of the production of VH-mode discharges is briefly examined to show that the observations are consistent with expectations from the $\mathrm{E} \times \mathrm{B}$ shear suppression hypothesis. New results are presented for discharges in which the time scale for the transition has been increased. These discharges exhibit changes in $E_{\mathrm{r}}$ prior to the transition and thus provide evidence that $E_{\mathrm{r}}$ causes the transition. Section III discusses the status of knowledge about the origin of $E_{\mathbf{r}}$ from the point of view of the radial force balance equation for toroidal equilibria. At this level of analysis, it is clear that the observed electric fields are 
a self-consistent result of various physical processes in the tokamak. Recent evidence is presented which indicates that the $\mathrm{V} \times \mathrm{B}$ term of the main ions controls the rapid change in $E_{\mathrm{r}}$ at the transition. Section IV contains a summary and conclusions. In this paper, the term "shear suppression" will mean suppression of turbulence by an $\mathrm{E} \times \mathrm{B}$ profile with non-zero first or second spatial derivatives. For the cases of interest, it is radial variation of $E_{\mathbf{r}}$ which produces this shear in $\mathrm{E} \times \mathrm{B}$.

\section{STUDIES OF L-H TRANSITION AND VH-MODE}

The spontaneous form of the L-H transition is generated in tokamaks by application of heating power above a required minimum called the power threshold. After the heating power is applied, the plasma passes through a phase of L-mode confinement during which temperatures and possibly densities rise monotonically with time. The classic signature marking the time of the transition is an abrupt and marked drop in the intensity of the $D_{\alpha}$ signal emitted from the plasma boundary. This signature is interpreted as indicating that the outflux of particles from the plasma decreases dramatically due to the formation of a transport barrier at the plasma edge which holds the particles in the plasma. The transport barrier is manifested as a dramatic steepening of the electron density $n_{\mathrm{e}}$, electron temperature $T_{\mathrm{e}}$ and ion temperature $T_{\mathrm{i}}$ profiles in a region with a width of the order of $1 \mathrm{~cm}$ just inside the last closed flux surface and a reduction of $n_{\mathrm{e}}$ on the open field lines [9]. These modified boundary conditions allow an improvement of confinement (thermal diffusivity) in the core of the plasma to occur so that the discharge attains a value of $\tau_{E}$ which is roughly twice its value in the $L-$ mode phase.

The phenomenology of the L-H transition in DIII-D is consistent with the expectations of the shear suppression hypothesis as has been discussed elsewhere [18-23]. Basically, at the L-H transition and in the same region of the plasma, there is a simultaneous formation of a negative "well" in $E_{\mathbf{r}}$ [9,18-21], a reduction in the level of electron density fluctuations $\tilde{n}[9,18,19,21-23]$ and an increase in electron and ion pressure gradients $[9,18,19]$. Due to the short time scale in which these changes occur, it has not been possible in previous work to unambiguously determine if changes in $E_{\mathbf{r}}$ occur prior to other changes, as might be expected from the shear suppression hypothesis. However, during the last year, experiments have been conducted in which the heating power was adjusted to be near the threshold value required to obtain the transition and this technique has slowed down the rate at which the transition occurs. Notably, significant changes in $E_{\mathbf{r}}$ are observed to occur prior to the transition [19,21], with $E_{\mathbf{r}}$ being determined directly from Langmuir probe measurements and by the standard spectroscopic techniques. These measurements of early changes in $E_{\mathrm{r}}$ are reminiscent of data reported several years ago [24] and are the strongest evidence for the causal role of $E_{\mathbf{r}}$ in the spontaneous L-H transition.

Figure 1 illustrates typical waveforms from these discharges with "slow" transitions. The time of the transition is inferred from Fig. 1(d) which displays the $\mathrm{D}_{\alpha}$ emission from the divertor target. The drop in the signal at $1480.4 \mathrm{~ms}$ is the classic signature of the transition. This time coincides with a marked reduction of the ion saturation current drawn by Langmuir probes in the divertor target. Figure 1(a) displays the values of $E_{\mathbf{r}}$ as obtained from two radii very near the plasma edge and shows that $E_{\mathbf{r}}$ starts becoming more negative and that the shear in $E_{\mathbf{r}}$ starts to increase several milliseconds before the transition. The level of electron density fluctuations is reduced abruptly at the transition as indicated by an FIR scattering system [Fig. 1(b)] and a microwave reflectometer system [Fig. 1(c)]. The signal from both instruments originates at the plasma edge. As indicated in Fig. 1(e), the electron pressure in the same region increases during the $\mathrm{H}$-mode with a time constant of several milliseconds. 
Under appropriate conditions, $\mathrm{H}-$ mode discharges evolve into a new regime of enhanced confinement, called the VH-mode, which exhibit $\tau_{\mathrm{E}}$ values $50 \%-100 \%$ larger than those obtained in $\mathrm{H}$-mode discharges. The transition to $\mathrm{VH}$-mode confinement occurs gradually over a period which is typically several tens of milliseconds $[14,15]$. During this time, the energy confinement time is increasing, the transport barrier gradually builds further into the plasma, there is an increase in the toroidal rotation velocity $v_{\phi}$ in the core of the plasma, and there is a simultaneous reduction of core microturbulence as determined via FIR scattering [25]. Heat transport coefficients in the plasma decrease during this time. The increasing value of $v_{\phi}$ indicates that shear in the $\mathrm{E} \times \mathrm{B}$ velocity is increasing in the region in which transport improves and the turbulence decreases. The changes in the $\mathrm{VH}$-mode occur in the core of the plasma rather than at the plasma boundary as for the $\mathrm{L}-\mathrm{H}$ transition. The shear suppression hypothesis predicts that if the core $E_{\mathrm{r}}$ shear is destroyed, then the VH-mode should be destroyed. This prediction has been verified with the aid of a magnetic perturbation which decreases the toroidal rotation $[18,19,26]$ with a corresponding decrease in the shear of $E_{\mathbf{r}}$. Simultaneously, the core turbulence levels rise, the local heat diffusion coefficients increase and the global confinement decreases. Thus, the hypothesis of $\mathrm{E} \times \mathrm{B}$ shear suppression of turbulence is capable of describing the phenomenology of the VH-mode as well as of the L-H transition in DIII-D.

\section{ORIGIN AND SHAPE OF RADIAL ELECTRIC FIELD}

A full understanding of the $\mathrm{L}-\mathrm{H}$ transition and of the $\mathrm{VH}-$ mode requires an understanding of the physics which controls the shape and magnitude of $E_{\mathrm{r}}$. There has been a large theoretical effort devoted to this issue which has led to the formulation of equations to describe $E_{\mathbf{r}}$ near the plasma boundary in toroidal geometry with neoclassical and anomalous effects included [27-29]. The experimental assessment of theory is very difficult due to the fact that many of the quantities of interest cannot be measured with existing diagnostic capability. The ideas which are being actively pursued can perhaps be divided into four categories:

1. Ion orbit loss [30-32]. Ions are preferentially lost from the plasma edge because ions in the loss cone intersect material surfaces. Thus, the plasma is charged up negatively.

2. Stringer spin-up [33,34]. A large poloidally asymmetric sink or source of particles overcomes the natural damping of poloidal rotation and allows a large 
poloidal rotation to develop. The relationship between poloidal rotation and $E_{\mathbf{r}}$ has not been specified in this model.

3. Pressure-gradient drive $[35,36]$. A toroidal equilibrium naturally develops a negative radial electric field to balance the pressure gradient of the ions. The large and negative $E_{\mathrm{r}}$ generated by the pressure gradient reduces transport through $\mathrm{E} \times \mathrm{B}$ shear. This feedback can cause a transport bifurcation.

4. Anomalous viscosity $[27,37,38,39]$ or turbulent Reynold's stress $[17,40]$. Transport of momentum can modify the average flow profile of the plasma. The relation between plasma velocity and $E_{\mathbf{r}}$ has been specified for these models.

On the experimental side, a convenient framework which is being used to examine the origin and shape of the $E_{\mathbf{r}}$ profile is the lowest order radial force balance equation

$$
E_{\mathrm{r}}=\left(Z_{\mathbf{i}} e n_{\mathbf{i}}\right)^{-1} \nabla p_{\mathbf{i}}-v_{\theta \mathbf{i}} \mathrm{B}_{\phi}+v_{\phi \mathbf{i}} \mathrm{B}_{\theta}
$$

where $i$ labels the ion species, $Z_{i}$ is the charge of the ion, $n_{i}$ is the ion density, $e$ is the electronic charge, $\mathrm{p}_{\mathrm{i}}$ is the ion pressure, $v_{\theta \mathrm{i}}$ and $v_{\phi \mathrm{i}}$ are, respectively, the poloidal and toroidal rotation velocities and $B_{\theta}$ and $B_{\phi}$ are, respectively, the poloidal and toroidal magnetic fields. Although this equation hides a great deal of physics, it shows clearly that the plasma's $E_{\mathrm{r}}$ is a self-consistent solution which satisfies several physical processes. These include the sources and transport of toroidal momentum, poloidal momentum, heat and particles as well as the generation of the magnetic equilibrium. Although the sources of several of these quantities are fairly well understood, the transport mechanisms in tokamaks are not understood. Thus, radial transport of heat, particles and momentum is generally considered to be "anomalous". There are theoretical suggestions that neoclassical effects may be important for rotation at the edge of the plasma, particularly in $\mathrm{H}$-mode [30,31,38,41-43]. An underlying assumption of present research is that, for a given experimental situation, one term on the right hand side of the force balance equation dominates and is considered to be responsible for $E_{\mathrm{r}}$ and the present goal of experimental research is to determine which is the dominant term.

For the VH-mode in DIII-D, the dominant term controlling $E_{\mathbf{r}}$ is $v_{\phi \mathbf{i}} \mathrm{B}_{\theta}$. At this level of understanding, the $E_{\mathrm{r}}$ profile and its shape are controlled by the input of toroidal momentum from neutral beam injection, which is understood to be a classical process, and the transport of toroidal momentum, which is not understood and is considered to be an anomalous process.

Studies of L-H transition have generally been based on the premise that the main ions control the physics of the edge $E_{\mathbf{r}}$, and one research goal has been to determine whether it is a change of the pressure gradient or of the $\mathrm{V} \times \mathrm{B}$ term of the main ions which initiates the rapid change in $E_{\mathrm{r}}$ observed at the $\mathrm{L}-\mathrm{H}$ transition. Although direct measurements of the behavior of pressure gradient and $\mathrm{V} \times \mathrm{B}$ terms for the main ions are not available at the transition, the slow transitions discussed above provide strong evidence that the main ion pressure gradient does not control the change in $E_{\mathrm{r}}$ at the transition [19]. These conclusions are based on measurements which show that the gradient of $T_{\mathrm{i}}$ does not change sufficiently at the transition to account for the $E_{\mathbf{r}}$ change and measurements which show that there are no significant changes in the electron density prior to the transition [19].

More quantitative analysis of the discharge discussed in Fig. 1 supports this conclusion. For this discharge, $E_{\mathbf{r}}$ and $T_{\mathrm{i}}$ profiles are available at the plasma edge every $0.5 \mathrm{~ms}$ 
before and after the $\mathrm{L}-\mathrm{H}$ transition. These data are obtained from He II, which is present in the plasma as an impurity and these measurements are a diagnostic used to determine the $E_{\mathbf{r}}$ and $T_{\mathrm{i}}$ values for the main ion force balance equation. In addition, a sequence of profiles of $n_{\mathrm{e}}$ and $T_{\mathrm{e}}$ from Thomson scattering are available every $2 \mathrm{~ms}$ from $1475 \mathrm{~ms}$ to $1487 \mathrm{~ms}$, a range which brackets the $\mathrm{L}-\mathrm{H}$ transition. Under the assumption that the ratio of $n_{\mathrm{e}}$ to the main ion density $n_{\mathrm{i}}$ is constant through the region of interest, the measurements of $T_{\mathrm{i}}$ and $n_{\mathrm{e}}$ can be combined to make an indirect estimate of the main ion pressure gradient contribution to $E_{\mathbf{r}}$. This is so because the constant of proportionality between $n_{\mathrm{i}}$ and $n_{\mathrm{e}}$ cancels out in the pressure gradient term of the force balance equation for the main ions.

Figure 1(a) demonstrates that there is a large change in the edge $E_{\mathbf{r}}$ between $1475 \mathrm{~ms}$, the time of the first Thomson laser pulse during the time of interest, and $1481 \mathrm{~ms}$, the time of the first Thomson laser pulse after the transition. Figure 2 is a comparison at these two times of the full $E_{\mathbf{r}}$ profiles and of the estimate for the pressure gradient term in the main ion force balance equation. These data show that although there is a profound change in $E_{\mathrm{r}}$ from 1475 to $1481 \mathrm{~ms}$, there is only a very modest change in the estimate for the main ion pressure gradient term. Thus, it must be concluded that either changes in the main ion pressure gradient do not cause the change in $E_{\mathrm{r}}$ across this transition or that the gradient of $n_{\mathrm{i}}$ changes drastically between these two times with little change occurring in the gradient of $n_{\mathrm{e}}$. The second possibility appears unlikely, particularly because simple estimates suggest that $n_{\mathrm{i}}$ may have to go negative to provide the required pressure gradient.

The lowest order force balance equation is expected to be valid for timescales much shorter than $1 \mathrm{~ms}$. Thus, the estimate of the main ion pressure gradient term developed above can be used to make an estimate of the temporal behavior of the $\mathrm{V} \times \mathrm{B}$ component of the main ion force balance equation at the $\mathrm{L}-\mathrm{H}$ transition. Figure 3(a) shows the time history for the total radial electric field $E_{\mathrm{r}}$, obtained at the location where the most negative value of $E_{\mathbf{r}}$ is observed in the $\mathrm{H}$-mode. At the same location, Fig. 3(b) shows the temporal history of $\left(e n_{e}\right)^{-1}$ $\nabla\left(\mathrm{T}_{\mathrm{i}} \mathrm{n}_{\mathrm{e}}\right)$. There is very little change in this term across the transition (1480.4 ms) with the pressure gradient term subsequently becoming significantly more negative. Figure 3 (c)shows the difference of $E_{\mathbf{r}}$ and the pressure gradient term and is an estimate of the temporal behavior of the $V \times B$ term of the main ions at the L-H transition. With the
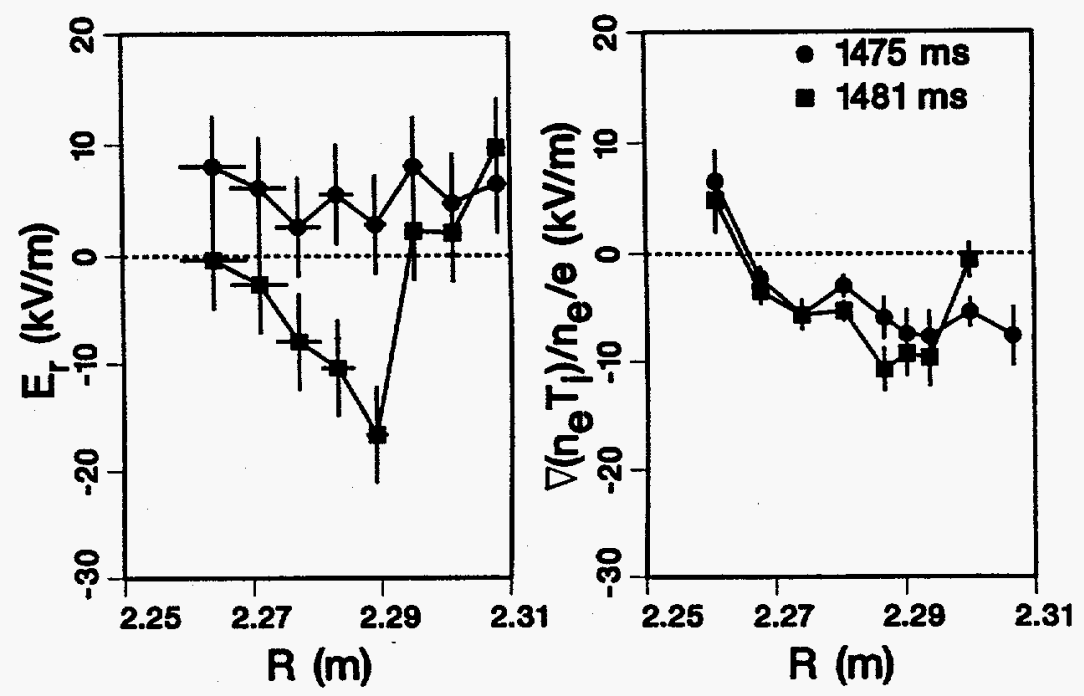

Fig. 2. Profiles at midplane of terms in force balance equation. Data for $1475 \mathrm{~ms}$ obtained in L-mode and data for $1481 \mathrm{~ms}$ obtained within one $\mathrm{ms}$ after transition. (a) $E_{\mathrm{r}}$ (obtained from He II force balance equation) shows significant changes between 1475 and $1481 \mathrm{~ms}$; (b) Estimate of main ion pressure gradient term, based on assumption that $n_{\mathrm{e}}$ and $n_{\mathrm{i}}$ profiles have similar shapes, shows little change between 1475 and $1481 \mathrm{~ms}$. 
caveats already discussed, these data indicate that the primary reason that $E_{\mathbf{r}}$ becomes more negative at the transition is that the $\mathrm{V} \times \mathrm{B}$ term of the main ions rapidly becomes less positive. The data suggest that the $\mathrm{V} \times \mathrm{B}$ term changes sign at the transition, but such a conclusion is perhaps premature until more data are examined. Figure 3(c) also suggests that as the $\mathrm{H}$-mode develops the main ion $\mathrm{V} \times \mathrm{B}$ term tends to go more positive again. For the claims made here, it is crucial that the relative timing accuracy of the Thomson Scattering and charge exchange recombination spectroscopy (CER) systems be better than about one millisecond. The absolute timing system accuracy of the CER system is good to within $0.1-0.2$ milliseconds and the accuracy of the Thomson system is even better.

These inferences about the $\mathrm{V} \times \mathrm{B}$ term of the main ions are consistent with direct measurements of the main ions in other discharges. The positive value for the $\mathrm{V} \times \mathrm{B}$ term of the main ions has been confirmed with direct measurements of the main ions in discharges with fast $\mathrm{L}-\mathrm{H}$ transitions [43]. For those discharges, it is known that the $\mathrm{V} \times \mathrm{B}$ term of the main ions is always positive except for a window of $\pm 3.5 \mathrm{~ms}$ around the time of the transition itself. Unfortunately, due to technical limitations associated with the measurements, data for the main ions across the transition are not available. Thus, there are no direct measurements of changes in the main ion $\mathrm{V} \times \mathrm{B}$ term. The direct measurements do show that $3.5 \mathrm{~ms}$ after the $\mathrm{L}-\mathrm{H}$ transition, the pressure gradient of the main ions is the dominant term in the force balance and causes the negative $E_{\mathrm{r}}$.

At various times in the $\mathrm{H}$-mode, all of the terms of the main ion force balance equation are important in controlling the negative $E_{\mathrm{r}}$ observed in the transport barrier in DIII-D. At the time of the transition itself, the $\mathrm{V} \times \mathrm{B}$ term appears to be important, indicating that the sources and transport of poloidal and, perhaps, toroidal momentum are important processes. Of these quantities, only the source of toroidal momentum in neutral-beam heated discharges can be considered well understood at this time. Shortly after the transition the pressure gradient becomes dominant and thus the edge $E_{\mathbf{r}}$ profile is controlled by the sources of heat and particles, which are understood as classical processes, and the transport mechanisms of heat and particles which are considered to be anomalous.

\section{SUMMARY AND CONCLUSIONS}

Sheared mass flows are of general interest in fluid mechanics because of their role in driving or subduing instabilities. For magnetized plasmas, there is a large body of theoretical and experimental evidence which indicates that sheared $\mathrm{E} \times \mathrm{B}$ flows, due primarily to sheared $E_{\mathbf{r}}$ fields, can stabilize a large class of instabilities found in laboratory plasmas. In the DIII-D tokamak, the phenomenology of two modes of improved confinement are consistent with the hypothesis that increased shear in $E_{\mathbf{r}}$ leads to a suppression of 
turbulence and improved confinement. In both the transition from $\mathrm{L}$-mode to $\mathrm{H}$-mode confinement and the transition from $\mathrm{H}$-mode to $\mathrm{VH}$-mode confinement, there is a strong spatial and temporal correlation between the increase of shear in $E_{\mathbf{r}}$, a reduction of $\tilde{n} / n$ which is evidence of a reduced level of turbulence and increased gradients in temperature and density profiles.

The mechanisms which control the magnitude and shape of $E_{\mathbf{r}}$ in a tokamak are poorly understood. The core $E_{\mathbf{r}}$ in the VH-mode in DIII-D is controlled by the plasma's toroidal rotation which is in turn controlled by the momentum input from neutral beam injection. Although, this source of momentum input is understood, the transport of toroidal momentum is also important in forming the $E_{\mathrm{r}}$ profile and is not well understood. The situation for the L-H transition is even more difficult, due to the rapidity with which the edge plasma evolves at the transition. However, the time scale for the transition has been increased by operating near the $\mathrm{H}$-mode power threshold. Evidence acquired from such transitions shows that the edge $E_{\mathrm{r}}$ starts to become more negative before changes are observed in turbulent quantities and before the transition occurs. In addition, the available data provides indirect but very strong evidence that it is the $V \times B$ rather than the pressure gradient term for the main ions which changes rapidly at the transition. Further studies are required to determine whether it is $v_{\phi}$ or $v_{\theta}$ of the main ions which changes at the transition.

\section{ACKNOWLEDGMENTS}

The results presented here are the result of the dedication and hard work of the entire DIII-D staff, including the technical, engineering, operations, computer and physics groups. This work was supported by the U.S. Department of Energy under Contract Nos. DE-AC03-89ER51114，W-7405-ENG-48，DE-FG03-89ER51121，DE-FG03-86ER53225, DE-AC05-84OR21400, DE-FG02-91ER54109 and DE-AC03-76DP00789.

\section{REFERENCES}

[1] Tajima, T., etal., Phys. Fluids B 3, 938 (1991).

[2] Kuo, H.L. Phys. Fluids 6, 195 (1963).

[3] Biglari, H., Diamond, P.H., Terry, P.W., Phys. Fluids B 2, 1 (1990).

[4] Hassam, A.B., Comments Plasma Phys. Controlled Fusion 14, 275 (1991).

[5] Hamaguchi, S., Horton, W., Phys. Fluids B 4, 319 (1992).

[6] Carreras, B.A., etal., Phys. Fluids B 4, 3115 (1992).

[7] Ritz, Ch. P., etal., Phys. Rev. Lett. 65, 2543 (1990).

[8] Groebner, R.J., Phys. Fluids B 5, 2343 (1993).

[9] Burrell, K.H., et al., Plasma Phys. Contr. Fusion 36, A291 (1994).

[10] Wagner, F., etal., Phys. Rev. Lett. 49, 1408 (1982).

[11] Taylor, R.J., et al., Phys. Rev. Lett. 63, 2365 (1989).

[12] Jackson, G.L., et al., Phys. Fluids B 4, 2181 (1992).

[13] JET Team, Plasma Phys. Contr. Fusion 36, A23 (1994).

[14] Greenfield, C.M., et al., Plasma Phys. Contr. Fusion 35, B263 (1993).

[15] Osborne, T.H., etal., Plasma Phys. Contr. Fusion 36, A237 (1994).

[16] Diamond, P.H., etal., Phys. Rev. Lett. 72, 2565 (1994). 
[17] Diamond, P.H., and Kim, Y-B., Phys. Fluids B 3, 1626 (1991).

[18] Burrell, K.H., etal., Phys. Plasmas 1, 1536 (1994).

[19] Burrell, K.H., et al., "H-Mode and VH-Mode Confinement Improvement in DIII-D: Investigations of Turbulence, Local Transport and Active Control of the Shear in the $E \times B$ Flow," in Proc. 15th Intnl Conf. on Plasma Phys. and Contr. Fusion Research 1994 (International Atomic Energy Agency, Vienna) to be published.

[20] Gohil, P., et al., "The Parametric Dependence of the Spatial Structure of the Radial Electric Field at the Plasma Edge in the DIII-D Tokamak," in Proc. 21st Euro. Physical Society Conf. on Contr. Fusion and Plasma Phys., Montpellier, France, 1994 (EPS, Petit-Lancy, Switzerland) to be published.

[21] Moyer, R.A., to be published in Physics of Plasmas.

[22] Doyle, E.J., etal., (1993). Plasma Physics and Controlled Nuclear Fusion Research 1992 (International Atomic Energy Agency, Vienna, 1993), Vol. 1, p. 235.

[23] Rhodes, T.L., etal., Rev. Sci. Instrum. 63, 4599 (1992).

[24] Groebner, R.J., Burrell, K.H., Seraydarian, R.P., Phys. Rev. Lett. 64, 3015 (1990).

[25] Rettig, C.L., et al., Plasma Phys. Contr. Fusion 36, A207 (1994).

[26] La Haye, R.J., et al., in Local Transport Studies in Fusion Plasmas (Proc. Varenna Workshop, 1993) (Societa Italiana di Fisca, Bologna, 1994) ISPP-14 p. 283.

[27] Rozhansky, V., and Tendler, M., Phys. Fluids B 4, 1877 (1992).

[28] Hinton, F.L., and Kim, Y.-B., Nucl. Fusion 34, 899 (1994).

[29] Yushmanov, P.N., et al., Phys. Plasmas 1, 1583 (1994).

[30] Itoh, S.-I., and Itoh, K., Nucl. Fusion 29, 1031 (1989).

[31] Shaing, K.C., Crume, Jr., E.C., and Houlberg, W.A., Phys. Fluids B 2 , 1492 (1990).

[32] Tendler, M. etal., (1993). Plasma Physics and Controlled Nuclear Fusion Research 1992 (International Atomic Energy Agency, Vienna, 1993), Vol. 2, p. 243.

[33] Hassam, A.B., Antonsen, Jr., T.M., Drake, J.F., and Liu, C.S., Phys. Rev. Lett. 66, 309 (1991).

[34] McCarthy, D.R., etal., Phys. Fluids B 5, 1181 (1993).

[35] Hinton, F.L., and Staebler, G.M., Phys. Fluids B 5, 1281 (1993).

[36] Staebler, G.M., et al., Phys. Plasmas 1, 909 (1994).

[37] Staebler, G.M., and Dominguez, R.R., Nucl. Fusion 33, 77 (1993).

[38] Rozhansky, V., and Tendler, M., "Prospects for Improved Confinement in Hot Plasmas," in Proc. 21st Euro. Physical Society Conf. on Contr. Fusion and Plasma Phys., Montpellier, France, 1994 (EPS, Petit-Lancy, Switzerland) to be published.

[39] Itoh, S-I., et al., Plasma Phys. Contr. Fusion 36, A261 (1994).

[40] Carreras, B.A., et al., "Dynamics of L to H Bifurcation and Ion Pressure Gradient Evolution," to be published in Phys. Plasmas 1.

[41] Stacey, W.M., and Jackson, D.R., Phys. Plasmas 1, 909 (1994).

[42] Rogister, A., Phys. Plasmas 1, 619 (1994).

[43] Hinton, F.L., and Kim, Y.-B., "Poloidal Rotation in Tokamaks with Large Electric Field Gradients," General Atomics Report GA-A21600, April, 1994, to be published in Phys. of Plasmas.

[44] Kim, J., et al., Plasma Phys. Contr. Fusion 36, A183 (1994). 